\title{
Trends in discovery of new drugs for tuberculosis therapy
}

\author{
Giovanna Riccardi and Maria Rosalia Pasca
}

After the introduction of isoniazid and rifampicin, the second one discovered in the Lepetit Research Laboratories (Milan, Italy), under the supervision of Professor Piero Sensi, tuberculosis (TB) was considered an illness of the past. Unfortunately, this infectious disease is still a global health fear, due to the multidrug-resistant Mycobacterium tuberculosis and extensively circulating drug-resistant strains, as well as the unrecognized TB transmission, especially in regions with high HIV incidence. In the last few years, new antitubercular molecules appeared on the horizon both in preclinical and clinical stage of evaluation. In this review, we focus on a few of them and on their mechanism of action. Two new promising drug targets, DprE1 and MmpL3, are also discussed.

The Journal of Antibiotics (2014) 67, 655-659; doi:10.1038/ja.2014.109; published online 6 August 2014

\section{TUBERCULOSIS CONTINUES TO BE A SERIOUS GLOBAL HEALTH PROBLEM}

Tuberculosis (TB), an old disease considered tied to the past, started again capturing the experts' attention in the early 1990s for different reasons, such as the HIV/AIDS pandemic and the appearance of Mycobacterium tuberculosis drug-resistant strains. ${ }^{1}$

In 2011, about nine million people were affected by TB and 1.4 million died, mostly in Asia (60\% of cases) and Africa $(24 \%){ }^{2}$ Multidrug-resistant TB (MDR-TB) is also a worrying public health issue in Eastern Europe, where the reported prevalence of MDR-TB ranges from 18 to $35 \%$ in new $\mathrm{TB}$ cases. $^{3}$

Generally, TB therapy is based on the combination of four drugs, rifampicin, isoniazid, ethambutol (EMB) and pyrazinamide (PZA) for 2 months, followed by rifampicin and isoniazid for 4 months. ${ }^{4}$ Unfortunately, poor patient compliance, as well as inadequate health care specialized structures, favored the selection of MDR-TB that requires at least 20 months of treatment with second line drugs (fluoroquinolones, amikacin, kanamycin and capreomycin), that are more toxic and less efficient, with cure rates in the range of $60-75 \% .^{5}$

MDR-TB are strains resistant to the most potent first-line drugs, rifampicin and isoniazid. In 2012, 450000 people developed MDR-TB in the world. It is estimated that about $9.6 \%$ of these cases were extensively-drug-resistant (XDR-TB), showing additional resistance to at least one fluoroquinolone and one injectable drug (amikacin, kanamycin or capreomycin). ${ }^{4,6}$ For patients affected by XDR-TB, the therapeutic efficacy is quite limited.

Recently, a few reports have claimed about the emergence of 'totally drug-resistant TB' with no chance of cure. ${ }^{7-9}$ However, there is a need to come to an agreement on the definition of these strains primarily in terms of severity. ${ }^{10}$
Therefore, new antitubercular drugs, as well as novel TB targets, are urgently required to overcome the problem of drug resistance and to finally eradicate TB.

\section{FOUR PIONEER DRUGS}

Pyridine-4-carboxy hydrazide (isonicotinyl hydrazide, INH, Figure 1a) was at the same time discovered in 1952 by three different pharmaceutical companies: Hoffmann LaRoche (Nutley, NJ, USA), ER Squibb \& Sons (Princeton, NJ, USA) and BAYER (leverkusen, Germany). This molecule saved many human lives and was defined 'magic drug' by the media. After many decades, INH still continues to be on the frontline in the battle against TB. ${ }^{11}$

It is widely accepted that INH is a prodrug that is activated by the M. tuberculosis catalase-peroxidase KatG enzyme to form an adduct with NAD. After that, this INH-NAD complex inhibits the NADHdependent enoyl-ACP reductase (encoded by inhA gene) of the fatty acid synthase type II system, involved in mycolic acid biosynthesis. This inhibition causes accumulation of long-chain fatty acids and cell death. ${ }^{12}$ Presently, about $70-80 \%$ of INH resistance in M. tuberculosis clinical isolates is attributed to mutations in the kat $G$ and $i n h A$ genes, respectively. ${ }^{11}$

As prodrug, INH activity is greatly influenced by mutations in $k a t G$ gene; consequently, a reasonable way to bypass this mechanism of resistance is designing drugs that target specifically InhA enzyme, without KatG activation. In this context, triclosan has been shown to specifically inhibit InhA. Unfortunately, its use as antitubercular was not successful because of its sub-optimal bioavailability. ${ }^{13}$ Using a structure-based drug design approach, a series of triclosan derivatives was synthetized. ${ }^{14}$ It is noteworthy that these derivatives demonstrated to be effective against $M$. tuberculosis INH-resistant laboratory and clinical strains. The best inhibitor had an MIC of 
a<smiles>NNC(=O)c1ccncc1</smiles>

b

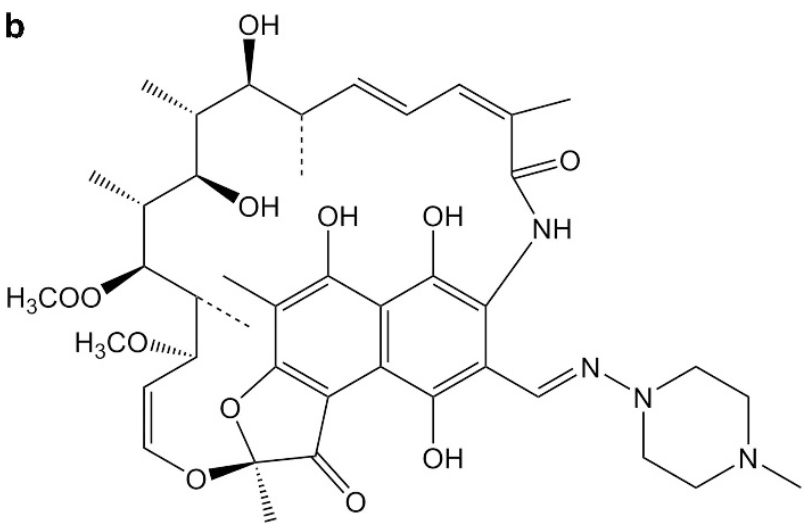

Figure 1 Chemical structure of Isoniazid (a) and Rifampicin (b).

$4.7 \mu \mathrm{g} \mathrm{ml}^{-1}$, which represents a tenfold improvement compared with the activity of triclosan, but clearly less potent than INH with an MIC of $50 \mathrm{ng} \mathrm{ml}^{-1} \cdot{ }^{14}$ Several scientists are searching for new antituberculars targeting InhA, without KatG activation and effective like INH. This is a difficult task as INH is very potent. Indeed, recently, new InhA inhibitors were synthetized, but their effectiveness was not as good as INH. ${ }^{15}$

In the last months, the pyridomycin, a natural compound produced by Dactylosporangium fulvum with specific 'cidal' activity against mycobacteria, was demonstrated to target InhA. ${ }^{16}$ Moreover, biochemical and structural approaches showed that pyridomycin inhibits InhA directly as a competitive inhibitor of the NADHbinding site, without activation of KatG. Interestingly, many of the $M$. tuberculosis INH-resistant clinical isolates are sensitive to pyridomycin, strongly underlining the efficacy of this drug. ${ }^{16}$

PZA, an analog of nicotinamide, is a prodrug, requiring the activation to pyrazinoic acid by the bacterial pyrazinamidase, coded by $p n c A$ gene. ${ }^{17}$ Recently, RpsA was described as PZA cellular target. ${ }^{18}$ Infact, the overexpression of the wild-type RpsA conferred resistance in M. tuberculosis. Moreover, some PZA-resistant M. tuberculosis strains without $p n c A$ mutations showed mutations in rpsA. RpsA is essential for translation and its $\mathrm{C}$ terminus is also involved in transtranslation. ${ }^{19}$ This last process is dispensable during active growth conditions, but it is also required for stress survival and pathogenesis in some bacteria. When the activated form pyrazinoic acid binds to RpsA, it prevents its binding to messenger RNA. PZA inhibition of the trans-translation may consequently interfere with survival under stressful, nonreplicating conditions in M. tuberculosis.

This behavior could explain how diverse stress conditions, such as starvation, acid $\mathrm{pH}$ and hypoxia, can potentiate PZA activity. ${ }^{18,20-22}$

$\mathrm{EMB}$ is involved in the inhibition of the polymerization of arabinogalactan, an important component of the mycobacterial cell wall. ${ }^{23}$ EMB interferes with the utilization of the arabinose donor by inhibiting either arabinosyl-transferase enzymatic activity or the formation of an arabinose acceptor in mycobacteria. ${ }^{23}$ The embCAB operon has been implicated in EMB resistance in M. tuberculosis. ${ }^{24}$
It is important to underline that EMB affects the same pathway blocked by the new class of drugs, benzothiazinones, even if not at the same metabolic step (see below).

The rifampicins are a group of antibacterial drugs which includes several derivatives like rifampicin, rifapentine, rifabutin and rifalazil. They target the beta RNA polymerase subunit, thus preventing transcription; resistance in $M$. tuberculosis is generally mediated by mutations mapping in an 81-bp region of the $r p o B$ gene, encoding the corresponding target. ${ }^{25}$

Rifampicin (Figure 1b) and isoniazid are the most widely used first-line molecules for TB therapy in combination with other agents. The introduction of rifampicin reduced the treatment of TB from 9 to 12 months to 6 months for active disease and from 9 to 3 months for latent infection, a peculiarity of the pathogen to become dormant in the host, in a nonreplicating condition. ${ }^{11}$ It is noteworthy that rifampicins are among the few drugs that can kill nonreplicating M. tuberculosis strains.

Rifampicin was developed in the Lepetit Research Laboratories (Milan, Italy), under the supervision of Professor Piero Sensi, as part of an extensive program of chemical modification of the rifamycins, the natural metabolites of Amycolatopsis mediterranei. ${ }^{26}$

As $r p o B$ is an essential gene and RNA polymerase a proven suitable target for anti-TB and antibacterial therapy, it seems reasonable to search for new RNA polymerase inhibitors that bind the same target, but differently from the rifamycin binding site. ${ }^{27}$

In 1989 Professor Piero Sensi wrote: 'In the last two decades, no new major anti-TB drug has been developed. Although dramatic improvements in chemotherapy for TB have been achieved through careful studies of drug regimens, there is still a need for new agents that are highly active. The antimycobacterial drugs used at present in therapy for TB were obtained by either blind screening or chemical modification of active compounds. Other approaches based on the knowledge of the biochemistry of the mycobacterial cell should be tried. Certain constituents of the cell, such as mycolic acids, arabinogalactan, peptidoglycan and mycobactins, may represent specific targets for new anti-TB drugs. ${ }^{28}$

As outstanding scientist Professor P. Sensi understood what TB scientists will realize later. It is not surprising that afterward many new compounds have been described to inhibit specific steps of mycolic acid or arabinogalactan biosynthesis. ${ }^{29}$

Even today, the scientists know very well that new TB drugs, as well as novel drug targets, are necessary in order: (1) to reduce the duration of therapy; (2) to be effective against MDR, XDR and perhaps totally drug-resistant TB strains; (3) to be effective against M. tuberculosis in its different physiological states including latent TB; (4) to show no antagonism with other anti-TB agents, as the therapy is based essentially on three different drugs; (5) to be compatible with anti-HIV therapy, as among the nine million TB patients around the world, $11-13 \%$ are HIV positive.

\section{TRENDS IN DISCOVERY OF NEW TB DRUGS}

After a long period of inactivity, the last few years have seen an increase in the number of new anti-TB drugs in the pipeline. As it can be seen in Figure 2, now there are an adequate number of drug candidates in Phase II and Phase III clinical trials as well as in preclinical development and in the stage of lead optimization. However, there is a worrying gap within Phase I that needs to be filled to have a constant delivery of molecules in case of failure of advanced drug candidates. ${ }^{30}$

Many molecules under clinical evaluation, such as fluoroquinolones, were developed to treat other infectious diseases and have now 

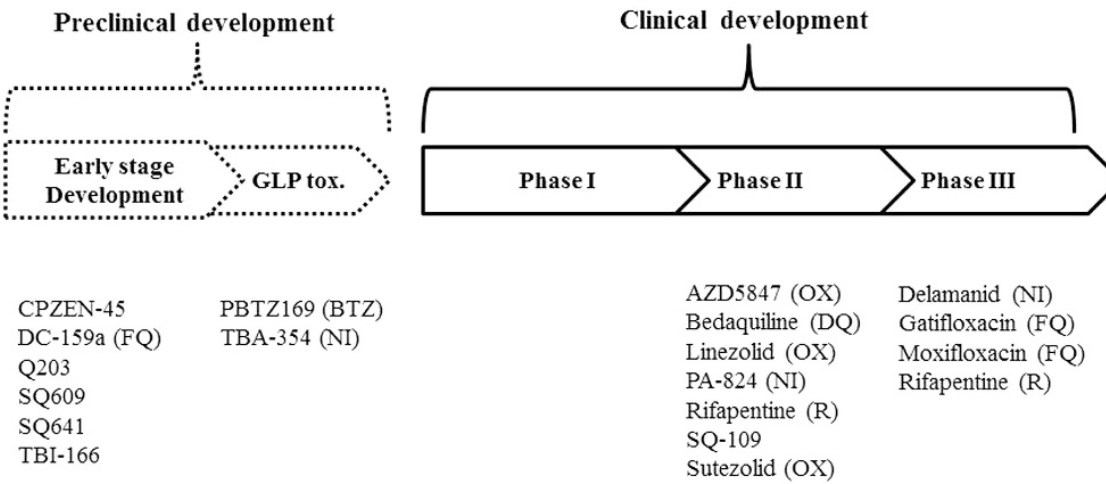

Figure 2 Current global drug pipeline (modified by www.newtbdrugs.org). FQ, fluoroquinolone; BTZ, benzothiazinone; NI, nitroimidazole; OX, oxazolidinone; $\mathrm{DQ}$, diarylquinoline; $\mathrm{R}$, rifamycin.

been repurposed for TB treatment, while other drugs were specifically discovered for TB treatment with activity against MDR and XDR-TB strains.

In this mini-review, we will focus only on a few new drugs that are in different stages of development as well as on a few 'hot' TB targets. Several comprehensive and updated reviews on this topic are available. ${ }^{30-32}$

Bedaquiline, a diarylquinoline, was approved by the FDA (Food and Drug Administration) in December 2012 as part of the combination therapy for the treatment of adult patients affected by MDR-TB and it is now in phase II of clinical development (Figure 2). It can be considered the first drug approved by FDA for TB in the last 40 years. $^{33}$ The diarylquinoline came out from a phenotypic screening of chemical molecules against mycobacterial growth, while the corresponding target was identified through whole-genome sequencing of $M$. tuberculosis and $M$. smegmatis spontaneous mutants resistant to the compound. These mutants showed missense mutations in the atpE gene, encoding the $c$ subunit of ATP synthase, thus interfering with the energy production. ${ }^{34}$

The mechanism of action of bedaquiline is peculiar and constitutes an alternative approach to current antimycobacterial killing, by inhibiting ATP synthase; it is effective against both replicating and dormant M. tuberculosis strains. It is well-known that pulmonary lesions can contain both types of populations, which are difficult to eliminate with conventional antitubercular drugs, thus favoring the development of resistance. ${ }^{35}$

It has been published that the human mitochondrial ATP synthase is 20000 fold less sensitive to diarylquinoline than the mycobacterial one, thus validating it as an important drug target, despite the fact that ATP synthase is highly conserved between Prokarya and Eukarya. ${ }^{36}$

Although bedaquiline has been linked to an increased risk of inexplicable mortality and QT prolongation, it represents a great addition for the treatment of MDR- and XDR-TB strains, especially in areas of the world where TB is endemic. ${ }^{37}$

Two new nitroimidazoles, PA-824 and OPC67683 (renamed delamanid), are in Phase II and Phase III clinical development, respectively (Figure 2). They are both pro-drugs whose activation depends on a F420-deazaflavin-dependent nitroreductase (Ddn) present in $M$. tuberculosis. The active form of PA- 824 is the corresponding des-nitroimidazole molecule, which generates reactive nitrogen species like nitric oxide. ${ }^{38}$ The respiratory poisoning through nitric oxide release appears to be crucial for its anaerobic activity. ${ }^{39}$
PA-824 is active against both replicating and dormant mycobacteria and preclinical and clinical investigations, suggesting that it may contribute in the shortening of TB duration therapy. ${ }^{40}$

The mechanism of action of delamanid is to inhibit mycolic acid biosynthesis and has been linked to an increase in sputum-culture conversion among patients affected by MDR-TB. ${ }^{41}$ Moreover, it showed efficacy with acceptable toxicity as part of a MDR-TB regimen. ${ }^{42}$

Rifapentine is a semisynthetic cyclopentyl rifamycin derivative; it binds the $\beta$-subunit of RNA polymerase, like the rifampicin. ${ }^{43}$ Rifapentine is more effective against $M$. tuberculosis, both in vitro and in vivo and the MIC ranges from 0.02 to $0.06 \mu \mathrm{g} \mathrm{ml}^{-1}$. ${ }^{44}$ As expected, there is cross-resistance between rifamycin and rifapentine.

In 1998, the US FDA approved rifapentine $\left(10 \mathrm{mg} \mathrm{kg}^{-1}\right)$ for oral administration once or twice weekly for both active and latent TB treatment. Regarding the latent $\mathrm{TB}$, the use of rifapentine plus isoniazid for 3 months (once-weekly regimen) is supported by good clinical evidence. Unfortunately, the results are quite different for the treatment of active $\mathrm{TB}$, where the rifapentine is approved by the FDA at $600 \mathrm{mg}$ dose orally, twice weekly during the intensive phase of TB treatment ( 2 months), then once weekly during the continuation phase ( 4 months). ${ }^{45}$

Recent animal studies suggested that more frequent administration might cure active and latent TB in 3 months or less, but these results were not reproducible in human trials. Moreover, in animal sperimentation, the inhaled administration seemed to improve the antitubercular activity in the lung, but clinical data are not yet available. $^{44}$

SQ109, a 1,2-ethylenediamine $\left\{\mathrm{N}^{\prime}\right.$-(2-adamantyl)-N-[(2E)-3,7dimethylocta-2,6-dienyl] ethane-1,2-diamine $\}$ is in Phase II clinical trials (Figure 2). SQ109 is active against M. tuberculosis, sensitive, MDR- and XDR-TB clinical strains. ${ }^{46,47}$ It was found from a 63238 chemical library, which was designed around the active 1,2ethylenediamine pharmacophore of EMB, one of the first-line antiTB drugs, hoping to find an EMB-like compound with better MIC and toxicity values. Surprisingly, the chemical structures of EMB and SQ109 are very different, as well as their mechanism of action.

SQ109 targets MmpL3, an essential membrane transporter belonging to the resistance, nodulation and division (RND) family, whose role in mycobacteria is to transport the trehalose monomycolate into the envelope thus inhibiting mycolic acid biosynthesis. ${ }^{48} \mathrm{MmpL} 3$ has another essential function in $M$. tuberculosis that needs to acquire iron for its survival, being involved, together Rv0203 and MmpL11, in mycobacterial heme uptake. ${ }^{48}$ 
MmpL3 is considered one of the most hot targets because several other compounds, under preliminary investigation, have also been reported to inhibit it, like the adamantyl urea derivative AU1235, the pyrrole derivative BM212, the C215 (N-(2,4-dichlorobenzyl)-1-propyl-1H-benzo[d]imidazol-5-amine) and the indoleamides. ${ }^{49-52}$

As MmpL proteins belong to RND family, which includes several efflux transporters able to extrude out of the bacterial cell many substrates such as antibiotics, dyes and detergents, ${ }^{53}$ we may not exclude the fact that MmpL3 acts through an efflux mechanism with the above cited drugs. However, it has been shown that the BM212 activity against mycobacteria was not influenced by the presence of efflux inhibitors like reserpine and verapamil, ${ }^{50}$ suggesting that MmpL3 may represent the true target of this compound. Further, it has been recently reported that a BM212 analog displays good efficacy in acute murine model of TB infection. ${ }^{54}$

Interestingly, all the M. tuberculosis spontaneous mutants resistant to the MmpL3 inhibitors show mutations that are mapped in different positions along the mmpL3 gene, unlike other drugs where the resistant mutants display mutations in the same codon. Most probably, structural studies could help to understand how MmpL3 binds these different inhibitors.

As foreseen by Piero Sensi, ${ }^{28}$ mycolic acids are one of the most important components of the mycobacterial envelope; many key enzymes are involved in this biosynthetic pathway, and most of them have been shown to be potential, excellent drug targets. ${ }^{29}$ In this context MmpL3 appears as a 'hot TB target'.

Another hot TB target is the M. tuberculosis DprE1 enzyme that is a decaprenylphosphoryl-beta-D-ribose 2-epimerase, an essential enzyme involved, together DprE2, in the formation of the decaprenylphospho-arabinose, a precursor for the synthesis of the cell-wall arabinans. ${ }^{55,56}$ The Benzothiazinones (BTZs) were the first drugs discovered to target this enzyme. ${ }^{57}$ All M. tuberculosis BTZ-resistant mutants present a point mutation in a cysteine residue of DprE1 (Cys387). ${ }^{57}$ The BTZs have a nitro group and are characterized by a covalent bond with the target. ${ }^{58}$ In particular, the nitro group of the drug is converted to a nitroso group by DprE1 itself. ${ }^{58}$ The nitroso form of BTZ is then able to covalently bind the Cys387, causing the DprE1 inhibition. ${ }^{58}$

In a first evaluation for the clinical use of BTZs, 240 M. tuberculosis-sensitive and drug-resistant strains turned out to be sensitive to this drug. ${ }^{59}$ Moreover, the synergism of BTZs with other antitubercular drugs, such as rifampicin, isoniazid, EMB, bedaquiline, PA-824, moxifloxacin, meropenem with or without clavulanate and SQ109 was investigated. The better synergism was found with bedaquiline, suggesting a possible good combination therapy. ${ }^{60}$

A new series of piperazine-containing benzothiazinones (PBTZ) more potent, safer and efficient in zebrafish and mouse models of TB have been recently published. ${ }^{61}$ One of them, PBTZ169, is in the last phase of preclinical trials (Figure 2). PBTZ169 binds covalently to DprE1 like BTZs. Combination of PBTZ169 with other drugs was studied. PBTZ169 showed additive activity against M. tuberculosis in vitro except with bedaquiline where synergy was observed. A new combination with PBTZ169, bedaquiline and PZA was demonstrated to be more effective than the standard three drug treatment in a murine model of TB. ${ }^{61}$

In the last four years several new DprE1 inhibitors with the same mechanism of action of BTZs were discovered like dinitrobenzamides, nitroquinoxalines and the 377790 triazole molecule. ${ }^{51,62-63}$

Recently, new antitubercular compounds targeting DprE1 in a different way were published: TCA1 compound, the azaindoles and other molecules are under investigation. ${ }^{64-65}$ Interestingly, these compounds do not possess the active nitro moiety and the binding with the target is mediated by non-covalent interactions. The availability of crystal structures of DprE1 in combination with some inhibitors, ${ }^{58,64,66}$ as well as the preclinical studies, could help us understand the precise mechanism of action of these DpE1 inhibitors and choose the better one(s) for clinical development.

\section{SUMMARY THOUGHTS}

In these last few years a significant number of new antitubercular drugs entered preclinical and clinical trials and for most of them the mechanism of action is known.

Drug discovery research is a complex, lengthy and expensive process. Many aspects have to be faced; first, the effectiveness of a compound in vitro, ex vivo and in vivo. Second, the synergy with the other antituberculars already used in therapy. Last but not least, the availability of specific grants for this research.

Recently, FDA allowed the use of bedaquiline for the treatment of pulmonary TB in adults affected by MDR-TB strains; after several decades finally a new compound has been introduced. ${ }^{33}$ However, this must not stop TB drug discovery research because although some new anti-TB molecules are available, others are needed to face the increasing problem of drug resistance. Moreover, the only available vaccine is the 'old' BCG, even if some new ones are in clinical trials. ${ }^{67}$ Consequently, the spreading of M. tuberculosis MDR, XDR and TDR strains is too dangerous for public health and, presently, stopping the TB diffusion with an efficient therapy is the most attainable.

It is noteworthy that several new $\mathrm{TB}$ compounds have two common targets: DprE1 and MmpL3. Both enzymes are essential for cell-wall biosynthesis, thus confirming the importance of this pathway in killing of the pathogen. This finding is in agreement with Sensi's thought few decades ago that the route to fight TB is to block the cell-wall formation.

\section{CONFLICT OF INTEREST}

The authors declare no conflicts of interest.

\section{ACKNOWLEDGEMENTS}

We acknowledge the European Commission (VII Framework, contract no. 260872) for funding our research.

1 Lienhardt, C. et al. Global tuberculosis control: lessons learnt and future prospects Nat. Rev. Microbiol. 10, 407-416 (2012).

2 Paulson, T. Epidemiology: a mortal foe. Nature 502, S2-S3 (2013).

3 Post, F. A. et al. Multi-drug-resistant tuberculosis in HIV positive patients in Eastern Europe. J. Infect. 68, 259-263 (2013).

4 World Health Organization. WHO Global Tuberculosis Report 2013, http://www.who.int/ tdr/news/2013/global-TB-report/en/ (2013)

5 Lienhardt, C. et al. New drugs for the treatment of tuberculosis: needs, challenges, promise, and prospects for the future. J. Infect. Dis. 205, S241-S249 (2012).

6 Green, K. D. \& Garneau-Tsodikova, S. Resistance in tuberculosis: what do we know and where can we go? Front. Microbiol. 4, 208 (2013)

7 Sotgiu, G., Spanevello, A. \& Migliori, G. B. In vitro susceptibility testing and totally drug-resistant tuberculosis. Eur. Respir. J. 42, 292 (2013).

8 Udwadia, Z. F., Amale, R. A., Ajbani, K. K. \& Rodrigues, C. Totally drug-resistant tuberculosis in India. Clin. Infect. Dis. 54, 579-581 (2012).

9 Velayati, A. A. et al. Emergence of new forms of totally drug-resistant tuberculosis bacilli: super extensively drug-resistant tuberculosis or totally drug-resistant strains in Iran. Chest 136, 420-425 (2009).

10 Migliori, G. B. et al. Totally drug-resistant and extremely drug-resistant tuberculosis: the same disease? Clin. Infect. Dis. 54, 1379-1380 (2012).

11 Riccardi, G., Pasca, M. R. \& Buroni, S. Mycobacterium tuberculosis: drug resistance and future perspectives. Future Microbiol. 4, 597-614 (2009).

12 Vilchèze, C. \& Jacobs, W. R. Jr. The mechanism of isoniazid killing: clarity through the scope of genetics. Annu. Rev. Microbiol. 61, 35-50 (2007) 
13 Wang, L.-Q., Falany, C. N. \& James, M. O. Triclosan as a substrate and inhibitor of 3'-phosphoadenosine 5'-phosphosulfate-sulfotransferase and UDP-glucuronosyl transferase in human liver fractions. Drug Metab. Dispos. 32, 1162-1169 (2004).

14 Freundlich, J. S. et al. Triclosan derivatives: towards potent inhibitors of drug-sensitive and drug-resistant Mycobacterium tuberculosis. ChemMedChem 4, 241-248 (2009).

15 Matviiuk, T. et al. Synthesis of 3-heteryl substituted pyrrolidine-2,5-diones via catalytic Michael reaction and evaluation of their inhibitory activity against InhA and $\mathrm{MyCO}$ bacterium tuberculosis. Eur. J. Med. Chem. 71, 46-52 (2014).

16 Hartkoorn, R. C. et al. Towards a new tuberculosis drug: pyridomycin - nature's isoniazid. EMBO Mol. Med. 4, 1032-1042 (2012).

17 Scorpio, A. et al. Characterization of pncA mutations in pyrazinamide-resistant Mycobacterium tuberculosis. Antimicrob. Agents Chemother. 41, 540-543 (1997).

18 Shi, W. et al. Pyrazinamide inhibits trans-translation in Mycobacterium tuberculosis. Science 333, 1630-1632 (2011).

19 Saguy, M. et al. Ribosomal protein S1 influences trans-translation in vitro and in vivo. Nucleic Acids Res. 35, 2368-2376 (2007).

20 Zhang, Y. et al. Mode of action of pyrazinamide: disruption of Mycobacterium tuberculosis membrane transport and energetics by pyrazinoic acid. J. Antimicrob. Chemother. 52, 790-795 (2003).

21 McDermott, W. \& Tompsett, R. Activation of pyrazinamide and nicotinamide in acidic environments in vitro. Am. Rev. Tuberc. 70, 748-754 (1954).

22 McCune, R. M., Feldmann, F. M., Lambert, H. P. \& McDermott, W. Microbial persistence. I. The capacity of tubercle bacilli to survive sterilization in mouse tissues. J. Exp. Med. 123, 445-468 (1966).

23 Wolucka, B. A. Biosynthesis of D-arabinose in mycobacteria-a novel bacterial pathway with implications for antimycobacterial therapy. FEBS J. 275, 2691-2711 (2008).

24 Telenti, A. et al. The emb operon, a gene cluster of Mycobacterium tuberculosis involved in resistance to ethambutol. Nat. Med. 3, 567-570 (1997).

25 Telenti, A. et al. Detection of rifampicin-resistance mutations in Mycobacterium tuberculosis. Lancet 341, 647-650 (1993).

26 Sensi, P. History of the development of rifampin. Rev. Infect. Dis. 5, S402-S406 (1983).

27 Mukhopadhyay, J. et al. The RNA polymerase "switch region" is a target for inhibitors. Cell 135, 295-307 (2008).

28 Sensi, P. Approaches to the development of new antituberculosis drugs. Rev. Infect. Dis. 11, S467-S470 (1989).

29 North, E. J., Jackson, M. \& Lee, R. E. New approaches to target the mycolic acid biosynthesis pathway for the development of tuberculosis therapeutics. Curr. Pharm. Des. 20, 4357-4378 (2014)

30 Zumla, A., Nahid, P. \& Cole, S. T. Advances in the development of new tuberculosis drugs and treatment regimens. Nat. Rev. Drug Discov. 12, 388-404 (2013).

31 Palomino, J. C. \& Martin, A. Tuberculosis clinical trial update and the current anti-tuberculosis drug portfolio. Curr. Med. Chem. 20, 3785-3796 (2013).

32 Nunn, A., Phillips, P. P. \& Abubakar, I. Treatment of pulmonary tuberculosis. Curr. Opin. Pulm. Med. 19, 273-279 (2013).

33 Mahajan, R. Bedaquiline: First FDA-approved tuberculosis drug in 40 years. Int. J. Appl. Basic Med. Res. 3, 1-2 (2013).

34 Andries, K. et al. A diarylquinoline drug active on the ATP synthase of Mycobacterium tuberculosis. Science 307, 223-227 (2005).

35 Chan, B., Khadem, T. M. \& Brown, J. A review of tuberculosis: focus on bedaquiline. Am. J. Health Syst. Pharm. 70, 1984-1994 (2013).

36 Haagsma, A. C. et al. Selectivity of TMC207 towards mycobacterial ATP synthase compared with that towards the eukaryotic homologue. Antimicrob. Agents Chemother. 53, 1290-1292 (2009).

37 Chahine, E. B., Karaoui, L. R. \& Mansour, H. Bedaquiline: a novel diarylquinoline for multidrug-resistant tuberculosis. Ann. Pharmacother. 48, 107-115 (2014).

38 Singh, R. et al. PA-824 kills nonreplicating Mycobacterium tuberculosis by intracelIular NO release. Science 322, 1392-1395 (2008).

39 Manjunatha, U., Boshoff, H. I. \& Barry, C. E. The mechanism of action of PA-824 Novel insights from transcriptional profiling. Commun. Integr. Biol. 2, 215-218 (2009).

40 Diacon, A. H. et al. Early bactericidal activity and pharmacokinetics of PA-824 in smear-positive tuberculosis patients. Antimicrob. Agents Chemother. 54, 3402-3407 (2010).
41 Gler, M. T. et al. Delamanid for multidrug-resistant pulmonary tuberculosis. New Eng/. J. Med. 366, 2151-2160 (2012).

42 Zhang, Q. Liu, Y. Tang, S., Sha, W. \& Xiao, H. Clinical benefit of delamanid (OPC67683 ) in the treatment of multidrug-resistant tuberculosis patients in China. Cell Biochem. Biophys. 67, 957-963 (2013)

43 Munsiff, S. S., Kambili, C. \& Ahuja, S. D. Rifapentine for the treatment of pulmonary tuberculosis. Clin. Infect. Dis. 43, 1468-1475 (2006).

44 Chan, J. G., Bai, X. \& Traini, D. An update on the use of rifapentine for tuberculosis therapy. Expert Opin. Drug Deliv. 11, 421-431 (2014).

45 Dooley, K. E. et al. Safety and pharmacokinetics of escalating daily doses of the antituberculosis drug rifapentine in healthy volunteers. Clin. Pharmacol. Ther. 91, 881-888 (2012).

46 Tahlan, K. et al. SQ109 targets MmpL3, a membrane transporter of trehalose monomycolate involved in mycolic acid donation to the cell wall core of Mycobacterium tuberculosis. Antimicrob. Agents Chemother. 56, 1797-1809 (2012).

47 Sacksteder, K. A., Protopopova, M., Barry, C. E. 3rd, Andriesm, K. \& Nacy, C. A. Discovery and development of SQ109: a new antitubercular drug with a novel mechanism of action. Future Microbiol. 7, 823-837 (2012).

48 Owens, C. P. et al. The Mycobacterium tuberculosis secreted protein Rv0203 transfers heme to membrane proteins MmpL3 and MmpL11. J. Biol. Chem. 288, 21714-21728 (2013).

49 Grzegorzewicz, A. E. et al. Inhibition of mycolic acid transport across the Mycobacterium tuberculosis plasma membrane. Nat. Chem. Biol. 8, 334-341 (2012).

$50 \mathrm{La}$ Rosa, V. et al. MmpL3 is the cellular target of the antitubercular pyrrole derivative BM212. Antimicrob. Agents Chemother. 56, 324-331 (2012).

51 Stanley, S. A. et al. Identification of novel inhibitors of $M$. tuberculosis growth using whole cell based high-throughput screening. ACS Chem. Biol. 7, 1377-1384 (2012).

52 Lun, S. et al. Indoleamides are active against drug-resistant Mycobacterium tuberculosis. Nat. Commun. 4, 2907 (2013).

53 Blair, J. M. \& Piddock, L. J. Structure, function and inhibition of RND efflux pumps in Gram-negative bacteria: an update. Curr. Opin. Microbiol. 12, 512-519 (2009).

54 Poce, G. et al. Improved BM212 MmpL3 inhibitor analogue shows efficacy in acute murine model of tuberculosis infection. PLOS ONE 8, e56980 (2013).

55 Wolucka, B. A. Biosynthesis of D-arabinose in mycobacteria - a novel bacterial pathway with implications for antimycobacterial therapy. FEBS J. 275, 2691-2711 (2008).

56 Riccardi, G. et al. The DprE1 enzyme, one of the most vulnerable targets of Mycobacterium tuberculosis. Appl. Microbiol. Biotechnol. 97, 8841-8848 (2013).

57 Makarov, V. et al. Benzothiazinones kill Mycobacterium tuberculosis by blocking arabinan synthesis. Science 324, 801-804 (2009).

58 Neres, J. et al. Structural basis for benzothiazinone-mediated killing of Mycobacterium tuberculosis. Sci. Transl. Med. 4, 150ra121 (2012).

59 Pasca, M. R. et al. Clinical isolates of Mycobacterium tuberculosis in four European hospitals are uniformly susceptible to benzothiazinones. Antimicrob. Agents Chemother. 54, 1616-1618 (2010).

60 Lechartier, B., Hartkoorn, R. C. \& Cole, S. T. In vitro combination studies of benzothiazinone lead compound BTZO43 against Mycobacterium tuberculosis. Antimicrob. Agents Chemother. 56, 5790-5793 (2012).

61 Makarov, V. et al. Towards a new combination therapy for tuberculosis with next generation benzothiazinones. EMBO Mol. Med. 6, 372-383 (2014).

62 Christophe, T. et al. High content screening identifies decaprenyl-phosphoribose 2' epimerase as a target for intracellular antimycobacterial inhibitors. PLoS Pathog. 5, e1000645 (2009)

63 Magnet, S. et al. Leads for antitubercular compounds from kinase inhibitor library screens. Tuberculosis (Edinb) 90, 354-360 (2010).

64 Wang, F. et al. Identification of a small molecule with activity against drug-resistant and persistent tuberculosis. Proc. Natl Acad. Sci. USA 110, E2510-E2517 (2013).

65 Shirude, P. S. et al. Azaindoles: noncovalent DprE1 inhibitors from scaffold morphing efforts, kill Mycobacterium tuberculosis and are efficacious in vivo. J. Med. Chem. 56, 9701-9708 (2013).

66 Batt, S. M. et al. Structural basis of inhibition of Mycobacterium tuberculosis DprE1 by benzothiazinone inhibitors. Proc. Natl Acad. Sci. USA 109, 11354-11359 (2012).

67 Orme, I. M. Vaccine development for tuberculosis: current progress. Drugs $\mathbf{7 3}$ 1015-1024 (2013). 